\title{
Characterization of Screen Printed Ag-PDMS Flexible Electrode for Electrical Muscle Stimulation (EMS)
}

\author{
Siti Noorjannah Ibrahim, Farah Abdul Rahman, Syaza Rosli \\ International Islamic University Malaysia \\ Department of Electrical and Computer Engineering, Kulliyyah of Engineering, International Islamic \\ University Malaysia (IIUM), Jalan Gombak, 53100 Kuala Lumpur, Malaysia \\ e-mail: noorjannah@iium.edu.my
}

\begin{abstract}
Electrical Stimulation is vital for maintaining muscle tone and strength particularly in improving muscle conditions. However, commonly used electrodes for muscle stimulation are flat, solid and have a fixed curvature and therefore cannot conform to the surface of the body. In this paper, a newly developed silver (Ag)- Polydimethylsiloxane (PDMS) flexible electrode for electrical muscle stimulation was developed. The electrodes were fabricated using screen printing for both single and array designs. The Ag-PDMS composite was developed to allow electrode pattern to be transferred properly on the PDMS substrate because due to same material property. Subsequently, the Ag-PDMS sheet resistance (R) which related to the conductivity of the electrode, was characterized. The $R$ increases each time strain was given to the substrate $(0.1 \mathrm{~cm}$ in each test) with the initial value of $0.7 \Omega$ and $75 \Omega$ for single and array electrode respectively.To evaluate the functionalities of the electrodes, the Mechanomyogram (MMG) signals were measured by using an equipment called USBamp and a commercial Electrical Muscle Stimulator. Results show that the functionality of the fabricated single electrodes were comparable to the commercial one.
\end{abstract}

Keywords: EMS; MMS; stretchable electrode; Ag-PDMS; screen printing

\section{Introduction}

Electrical Muscle Stimulation (EMS) also known as Neuromuscular Electrical Stimulation (NMES) is the activity of muscle contraction by using electrical impulses. Its functions are to relax muscle spasm, increase local blood circulation and maintain as well as increase the range of motions. In order to undergo EMS, electrodes are needed because they provide interface function between body and the electronic circuit. Electrode acts as the transducer that converts signals between the electronic circuitry and the human body. For stimulating electrode, the electric current is sent from unit of stimulating electrode and delivered into the muscle causing a contraction.

Some limitations of the common EMS are: rigid shape as such they have limited flexibility, not able to follow curvature surface of nonconformal human body, and uncomfortable when stimulations are made for a long period of time. Moreover, this type of electrode can cause severe skin ulceration due to inappropriate positioning. These commercial electrodes can be costly because of the materials and the techniques used. Therefore, a flexible electrode using cheaper manufacturing technique and material such as polymer can be an alternative solution for this issue.

\subsection{Ag-PDMS}

Polymers such as PDMS are not as stiff as metal but not as soft as liquids. The mechanical properties of polymers are different from other types of materials. Several advantages of PDMS are cheap, biocompatible, transparent and has low auto fluorescence, hence PDMS is suitable to be used in the fabrication of stretchable electrode [1]. Although gold $(\mathrm{Au})$ and silver $(\mathrm{Ag})$ inks are costly compared to copper $(\mathrm{Cu})$ ink, $\mathrm{Cu}$ is easily oxidized in air and oxidized copper is poor conductance [2]. Hence, $\mathrm{Ag}$ has the benefits as electrode material due to its high conductivity,excellent durability and fast drying characteristics. Ag also can be mixed with polymer to create a new composite that is cheaper than pure Ag but still give comparable electrical properties.

The Scanning Electron Microscopy (SEM) image (Figure 1(a)) of Ag-PDMS cured composite with particles are seen to be in contact with each other and they were distributed 
uniformly in the substrate (PDMS) [3]. Studies showed that a flexible and stretchable circuit could have resistance that increased almost linearly from $0.85 \Omega$ to $4.48 \Omega$ when expanded to $100 \%$ due to first tensile strain [4]. After releasing the strain, the resistance has partially recovered and remained nearly constant upon to $50 \%$. Fifty stretching/releasing cycles in the strain range of $0-50 \%$ (Figure $1(b)$ ).

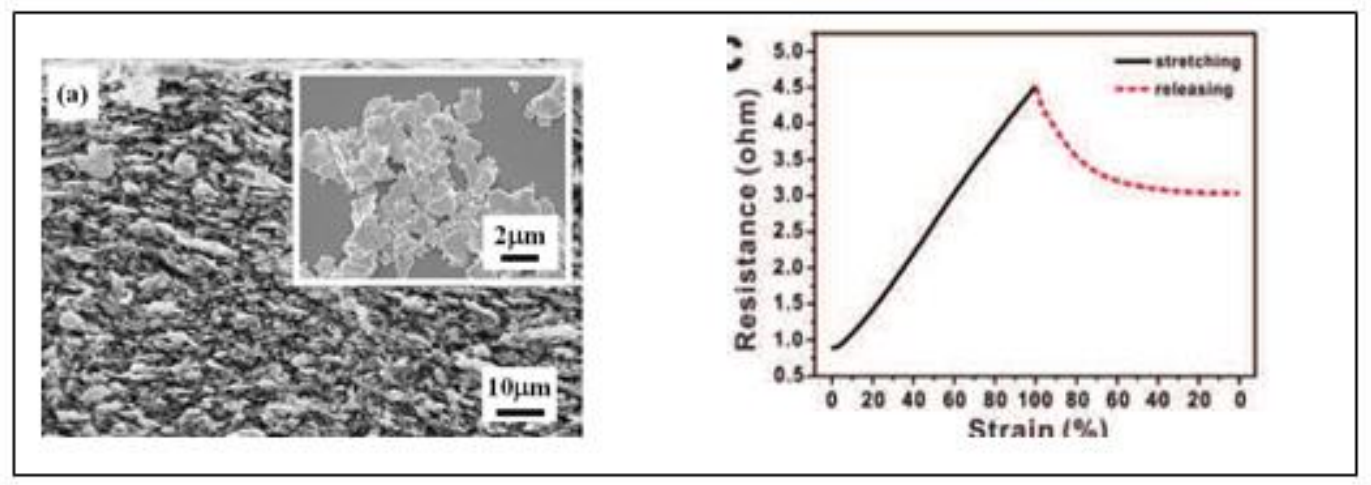

Figure 1. (a) SEM image of Ag-PDMS [3] (b) The resistance of Ag NWs/PDMS stretchable electrode when stretching remained nearly constant upon to $50 \%$ during 50 stretching/releasing cycles in the strain range of $0-50 \%$ [4].

Therefore, both PDMS and Ag have very interesting properties that can be manipulated in the development of stretchable electrode. Another study conducted in [5] also shown that AgPDMS can be used to produce low cost elastomeric stretchable printed circuit board (PCB). For this reason, the work presented here is about Ag-PDMS composite electrode constructed on PDMS substrate with two designs which is array and single design. Moreover, the work is compared to the $\mathrm{Ag}$ on OHP film in terms of their properties and functionalities. The OHP film chosen because of its transparent and flexible properties.

The stiffness of PDMS material can be easily adjusted by systematically adjusting the crosslinking process i.e., the lower the degree of PDMS chemical network cross-linking, the softer PDMS subtrate is [6]. PDMS stiffness may varied by changing the ratio of cross-linker to the base polymer. The common PDMS network ratio is 10:1 for low stiffness and certain degree of flexibility (to be stretched and bent) [6].

Here, we studied and characterized Ag-PDMS composite on PDMS substrate suitability to be used as EMS electrode. Two types of electrode were designed: the single and array electrodes. The former electrode (Figure 2(c)) has similar design to the commercial electrode while the latter electrode (Figure 2(e)) has wider size than former electrode. One advantage of array electrode for EMS is that it can cover a wider surface area hence can stimulate on individual muscle or small muscle. As a result, the stimulation process can be conducted in lesser time than using single electrode. From previous study, array electrode commonly used for Functional Electrical Stimulation (FES) because the user can switch to different array channel when stimulating which is to different type of small muscle or individual muscle [9][10]. The following subsections described the development process, electrical characterizations and functionality tests for the designed Ag-PDMS electrode.

\section{Research Method}

\subsection{Fabrication of Ag-PDMS electrode}

The flexible electrode for electrical muscle stimulation comprises of three different layers; the substrate (PDMS/OHP film) as the bottom layer, the conductive Ag/ Ag-PDMS paste as the intermediate layer and the Hydrogen Gel as the top layer that contacts to human skin (Figure 2(a)). The PDMS substrate was prepared using Slygard 184 Silicone Elastomer base and its curing agent with 10:1 ratio. Then, the PDMS mixture was degassed in a vacuum 
chamber. Note that, the degassing process is finished when the PDMS mixture is completely clear and transparent.

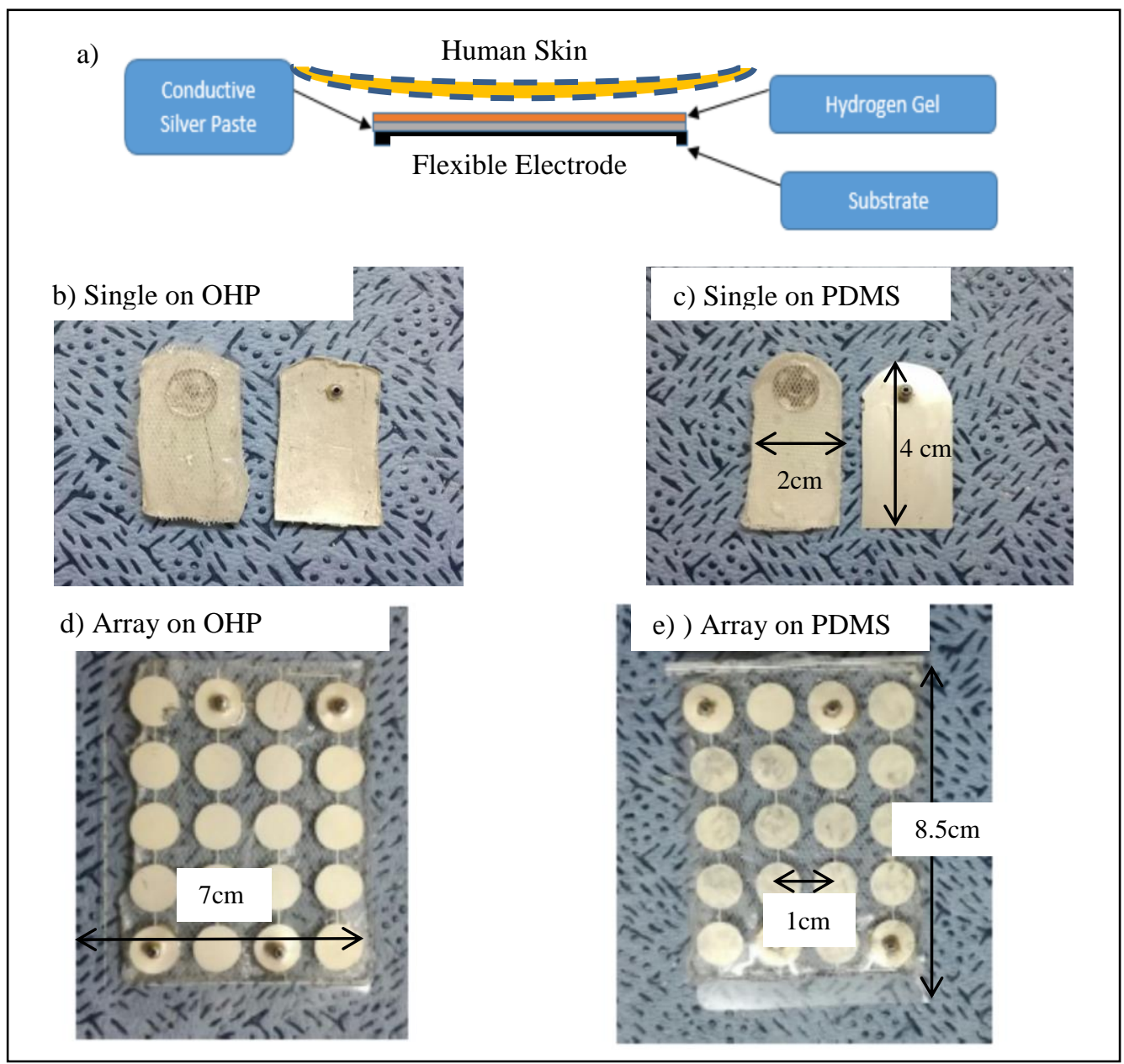

Figure 2 (a) Materials that construct the flexible electrode and types of flexible electrode on OHP and PDMS subtrates: (b) \& (c) Single Electrode and (d) \& (e) Array electrode.

To solidify, the PDMS material was left at room temperature for 24 hours. Meanwhile, to transfer patterns on the PDMS substrate, a newly developed composite made from Ag-PDMS paste was used.The composite was made by mixing PDMS material with conductive $\mathrm{Ag}$ polymer paste (Gwent Electronic Materials). The Ag-PDMS composite needs to be kept in a fridge at $4^{\circ} \mathrm{C}$ and must be degassed before usage. On the other hand, only Ag polymer paste was used as the electrode material on OHP film (Epson) substrate.

There were two designs studied in this paper; single and array electrodes (Figure 2(b)(e)); to compare electrical stimulation signals transmitted to the muscles. Before transferring the electrode patterns onto the substrates a pattern mesh was prepared on a polyester material (200 threads/inch) with Aluminum frame (Jujo Technology Resources). Firstly, the mesh was adjusted tightly on the holder and then, the substrate was placed under the screen (Figure 4(a)). The pattern mesh must adhered strongly onto the substrate and kept in flat position during the screen printing (pattern transfer) process. Subsequently, the Ag-PDMS paste was spread on the pattern mesh using a squeegee. To prevent the substrate from sticking to the mesh, it is important to make sure that the distance between them must be approximately $4 \mathrm{~mm}$. To dry the paste, the designed electrodes were baked in an oven at $150^{\circ} \mathrm{C}$ for 30 minutes. Finally, the top layer was covered with hydrogen gel so that the electrode can firmly attached to human skin. 


\subsection{Sheet Resistance Test on electrode}

The reason of this study is to investigate the effect of mechanical forces i.e., stretching and bending on sheet resistance. The sheet resistance is directly proportional to the resistivity; using equation $\rho=R \times S / L$ where $S$ is the strain given and $\mathrm{L}$ is the length of the substrate; which effect the conductivity of electrode [8].The adhesion ability between substrate and paste are very important especially for stretchable circuit while weak adhesion can lead to failure of the circuit after repeated force applied which is unacceptable for electrical device. For this experiment, the adhesion between them is crucial to ensure the effectiveness of electrical stimulation when place on the surface of body and the electrode can be used repeatedly. The electrode was wrapped around a $20 \mathrm{~mm}$ cylinder and the readings of the resistivity were taken before, during and after bending (Figure 4(b)) using a multimeter. Changes on the resistivity are expected, but what is more important is to observe how does the resistivity changes due to bending. It is also important to know the effect when the electrode is bent and reformed as well as to ensure the paste is still intact on the substrate.

\subsection{Mechanomyogram (MMG) Test}

After the fabrication processes were done, the electrode must be tested to evaluate its functions using commercial muscle stimulator. The biceps muscle was chosen in the testing (Figure 4(c)). The electrode from USBamp which is the equipment used to measure the MMG of the muscle, was placed next to the stimulated electrode as shown in Figure 4(d). The USBamp Simulink indicated the setup of the MMG. Once measurements were completed, the signals will be recorded and saved.

\section{Results and Analysis}

\subsection{Surface Roughness of the Ag-PDMS electrode}

The distribution of the Ag-PDMS composite paste on the PDMS and Ag on OHP film were obtained using microscope and Scanning Electron Microscopy (SEM). From the microscopic image, the Ag-PDMS electrode has coarser surface and uneven edge compared to the Ag electrode shown in Figure 5.The former electrode also has bigger Ag grain that the latter electrode. From the SEM image the Ag-PDMS, it can be observed that the Ag-PDMS composite distributed uniformly on PDMS and the particles are seen to be in contact with each other. However, there are some cavities/holes visible which might indicate the presence of PDMS material due to the its polymeric molecules size. This could also be inferred that some of the $\mathrm{Ag}$ (metal) and PDMS (Polymer) are detached from the composite due to either the composite was not mixed or degassed properly.

In contrary, the Ag particles are seen to be in contact with each other and the paste is distributed uniformly on the OHP film. The SEM image of the surface can be related to the resistivity of the electrode. The cavities due to detachment of Ag particles from the PDMS will affect electrode's resistivity. Low resistivity can be achieved if $\mathrm{Ag}$ is distributed evenly on the substrate. From the observation above, the Ag on OHP film electrode is expected to have low resistivity than Ag-PDMS on PDMS because the distribution of Ag on the OHP film is better than PDMS. 

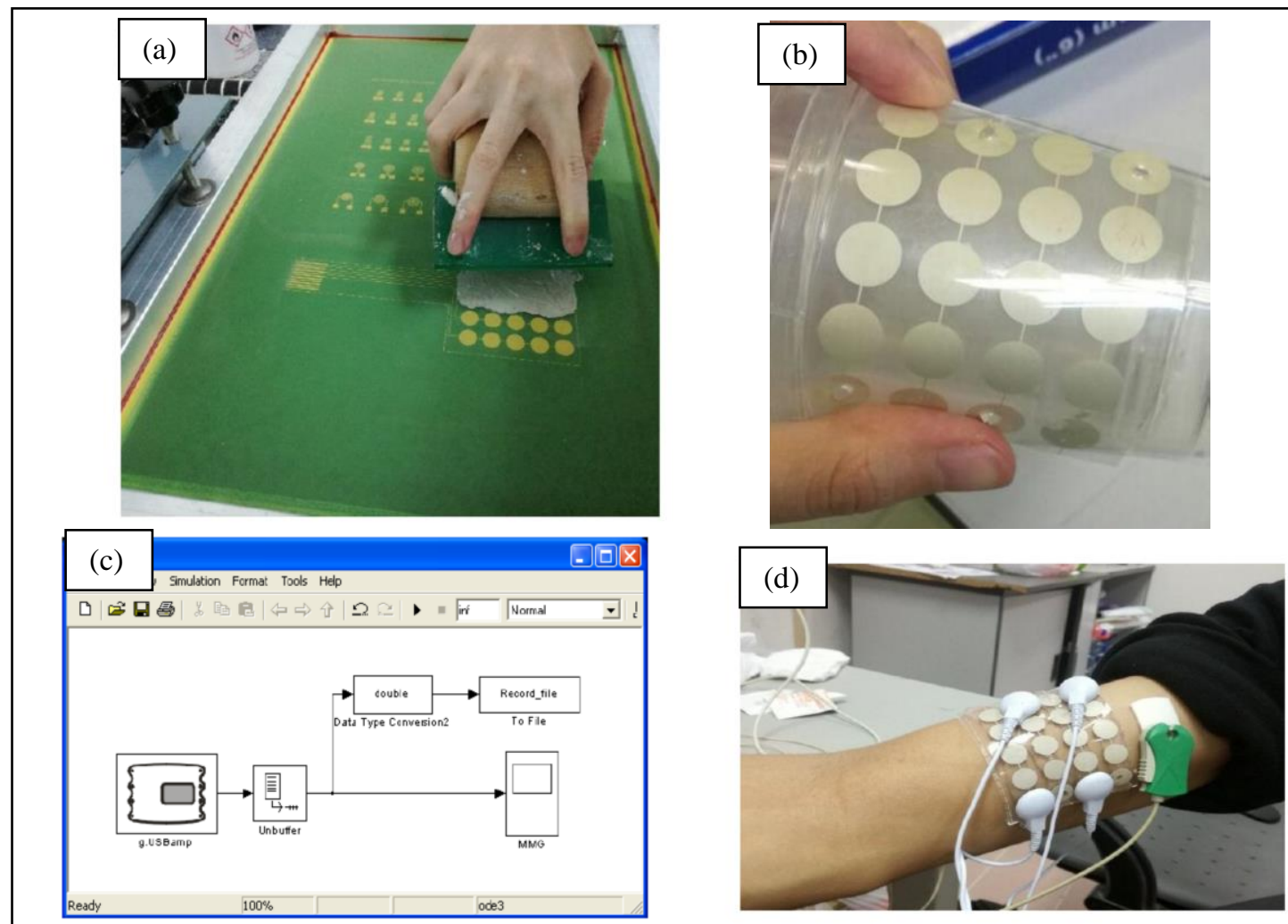

Figure 4.(a) Screen Printing Process. (b) Bending test on the electrode to observe its resistivy.

(c) The Simulink diagram of muscle measurement (USBamp). (d) Detection of muscle stimulation electrode using mechanomyogram (MMG).

In contrary, the Ag particles are seen to be in contact with each other and the paste is distributed uniformly on the OHP film. The SEM image of the surface can be related to the resistivity of the electrode. The cavities due to detachment of Ag particles from the PDMS will affect electrode's resistivity. Low resistivity can be achieved if $\mathrm{Ag}$ is distributed evenly on the substrate. From the observation above, the $\mathrm{Ag}$ on OHP film electrode is expected to have low resistivity than Ag-PDMS on PDMS because the distribution of Ag on the OHP film is better than PDMS.

\subsection{Ag-PDMS electrode sheet resistance}

Results in Figure 6 indicate the resistance of both single and array Ag-PDMS electrodes. The sheet resistiance $(R)$ increases each time strain was given to the substrate $(0.1 \mathrm{~cm}$ in each test) with the initial value of $0.7 \Omega$ and $75 \Omega$ for single and array electrode respectively. As expected, the sheet resistance of array electrode is higher than the single electrode, due to the multiple electrodes on a single substrate. The sheet resistance increases linearly with positive trends which means as more strain given to the substrates the conductivity of the electrode will be affected [8]. Ag-PDMS flexibility (allow stretch and bend) changes the conductive path of the electrode and therefore varied the resistivity. 


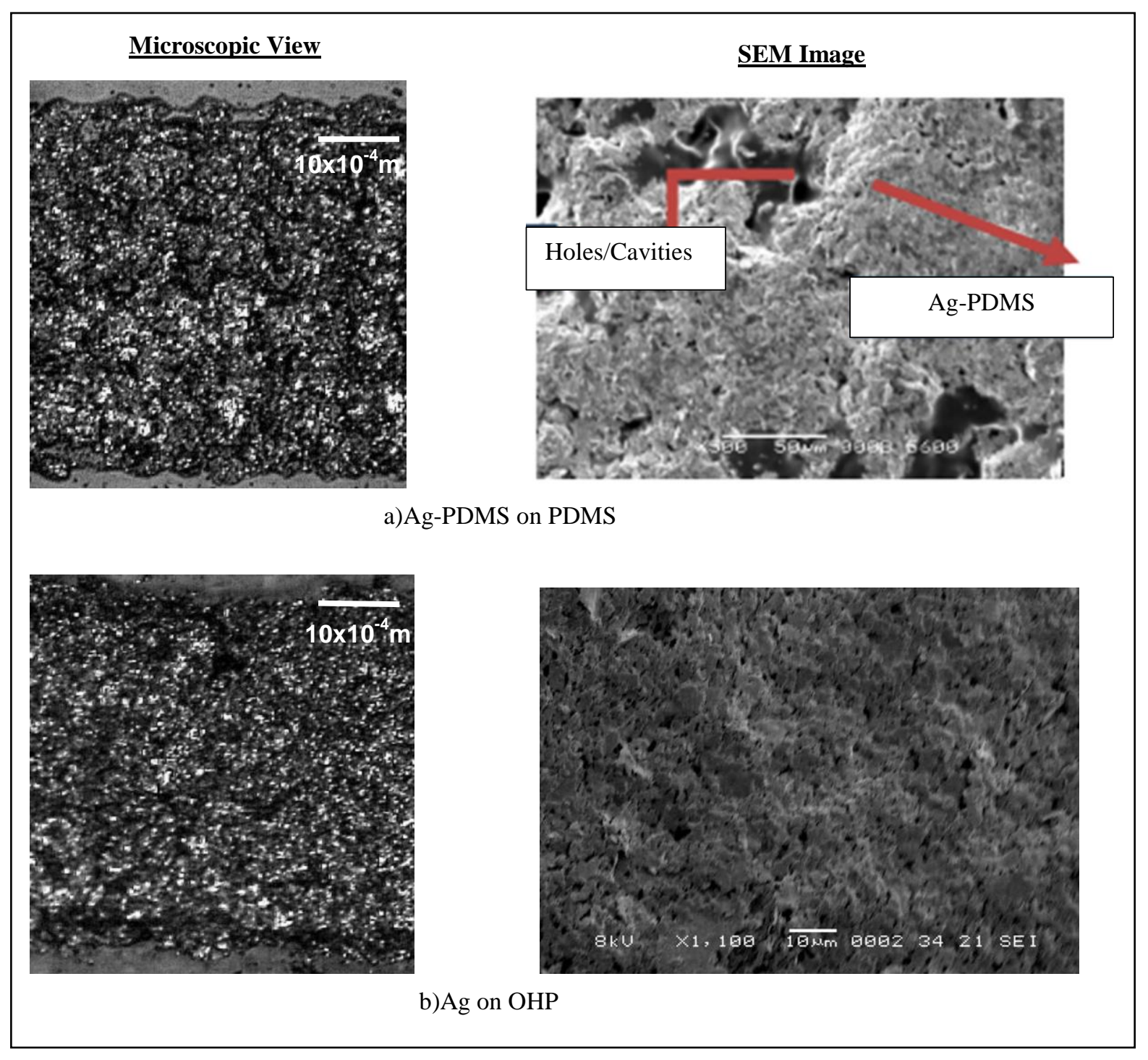

Figure 5. Microscopic and SEM images of Ag-PDMS and Ag on PDMS and OHP film substrates.

\subsection{Ag-PDMS electrode sheet resistance}

Results in Figure 6 indicate the resistance of both single and array Ag-PDMS electrodes. The sheet resistiance $(R)$ increases each time strain was given to the substrate $(0.1 \mathrm{~cm}$ in each test) with the initial value of $0.7 \Omega$ and $75 \Omega$ for single and array electrode respectively. As expected, the sheet resistance of array electrode is higher than the single electrode, due to the multiple electrodes on a single substrate. The sheet resistance increases linearly with positive trends which means as more strain given to the substrates the conductivity of the electrode will be affected [9]. Ag-PDMS flexibility (allow stretch and bend) changes the conductive path of the electrode and therefore varied the resistivity.

In the bending test, the sheet resistance of Ag-PDMS electrode in Table 1 increases during bending and slightly decreases when return to the actual position. On contrary, the sheet resistance values for $\mathrm{Ag}$ on OHP film electrode have no significant differences during and after the bending test with the average readings are $0.3 \Omega$ and $3.4 \Omega$ for single and array design respectively. It could be inferred that this effect could be contributed by the surface roughness 
and $\mathrm{Ag}$ distribution on OHP film is better that the Ag-PDMS (Figure 5(b)) that give strong adhesion on the substrate and resulted with consistent sheet resistance.

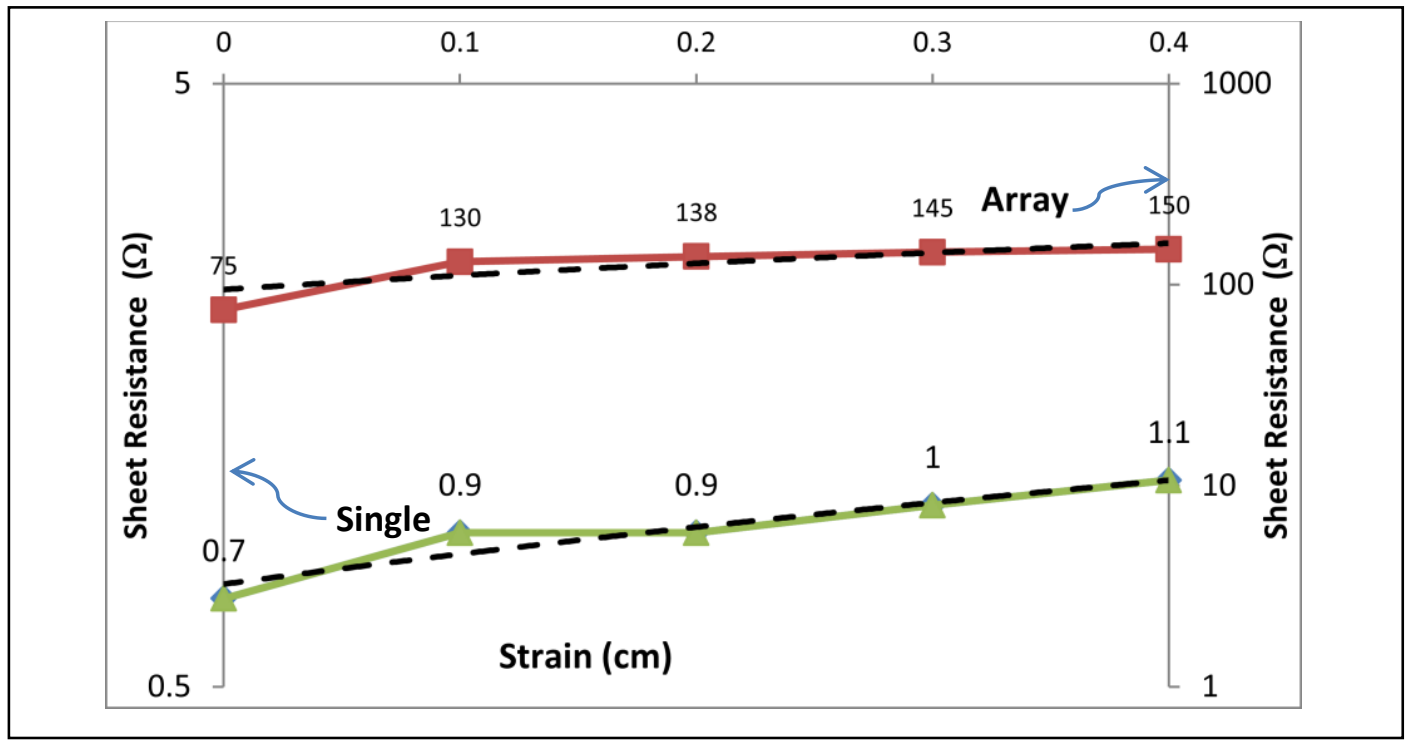

Figure 6. Sheet Resistance of the Ag-PDMS single and array electrodes.

Table 1 Sheet Resistance Reading when Bending Process

\begin{tabular}{lllllll}
\hline \multirow{2}{*}{ Type } & \multicolumn{2}{l}{ Before $(\mathrm{ohm})$} & \multicolumn{2}{l}{ During $(\mathrm{Ohm})$} & \multicolumn{2}{l}{ After (Ohm) } \\
\cline { 2 - 7 } & $\begin{array}{lllll}\text { Ag- } \\
\text { PDMS }\end{array}$ & Ag on OHP & $\begin{array}{l}\text { Ag- } \\
\text { PDMS }\end{array}$ & Ag on OHP & $\begin{array}{l}\text { Ag- } \\
\text { PDMS }\end{array}$ & $\begin{array}{l}\text { Ag on } \\
\text { OHP }\end{array}$ \\
\hline Single & 0.7 & 0.3 & 1.2 & 0.3 & 0.9 & 0.3 \\
Array & 80 & 3.4 & 130 & 3.5 & 120 & 3.4 \\
\hline
\end{tabular}

As the sheet resistance is a factor for resistivity $(\rho)$, the induced strain on sheet resistance would increase resistivity and therefore, when the conductive paths produced during the normal position are enlarged during bending, resistivity increases and conductivity decreases. Hence, the MMG signals transmitted from the muscle stimulator will be affected. From the observation, the $\mathrm{Ag}-\mathrm{OHP}$ film electrode gives lowest resistivity compact to the $\mathrm{Ag}$ PDMS electrode because it is less flexible (cannot stretch) than the latter. Overall, the resistivity of Ag- PDMS electrode is higher than Ag-OHP film which means the conductivity of Ag-PDMS electrode is lower than Ag-OHP film electrode. This is due to the presence of PDMS polymer in the Ag paste and also the mechanical charactierics of the substratret.

\subsection{Ag-PDMS electrode MMG signal Test}

The mechanomyogram (MMG) signal; the mechanical signal observable from the surface of a muscle whenever the muscle has contractions; are measured using the Ag-PDMS single electrode and then compared to the commercial single electrode. Results show that MMG signals from the Ag-PDMS electrode gave similar response to the commercial electrode ( Figure 12 and 13). This shows that the EMS impulses generated by the stimulator are delivered to the muscles through the fabricated electrode which acts as interface. The results prove that the fabricated Ag-PDMS single electrode is well functioning and comparable with the commercial electrical muscle stimulator electrode with the average peak of $7000 \mathrm{mV}-10000 \mathrm{mV}$ every $1 \mathrm{~ms}$. Between single electrode and array electrode, results indicate that the MMG peak signal of single electrode is much higher than array electrode for both substrates around $1500 \mathrm{mV}$ $2000 \mathrm{mV}$ every $1 \mathrm{~ms}$ ( Figure ). 
The results indicate that the electrical stimulus given to the specific muscle is smaller than the single array which can be useful if a continuous electrical stimulation is needed, for example in Functional Electrical Stimulation of stroke patient (FES) which can hinder muscle fatigueness where user can switch to different array channel when stimulating which is to stimulate different types of small muscle (Chen et al., 2007). However, noise level during muscle relaxation of array electrodes is higher than the single electrode which could affect signal readings at that point. Therefore, it is best to use single electrode for EMS rather than array electrode while the array electrode is suitable for user who wants low strength of contraction array electrode.

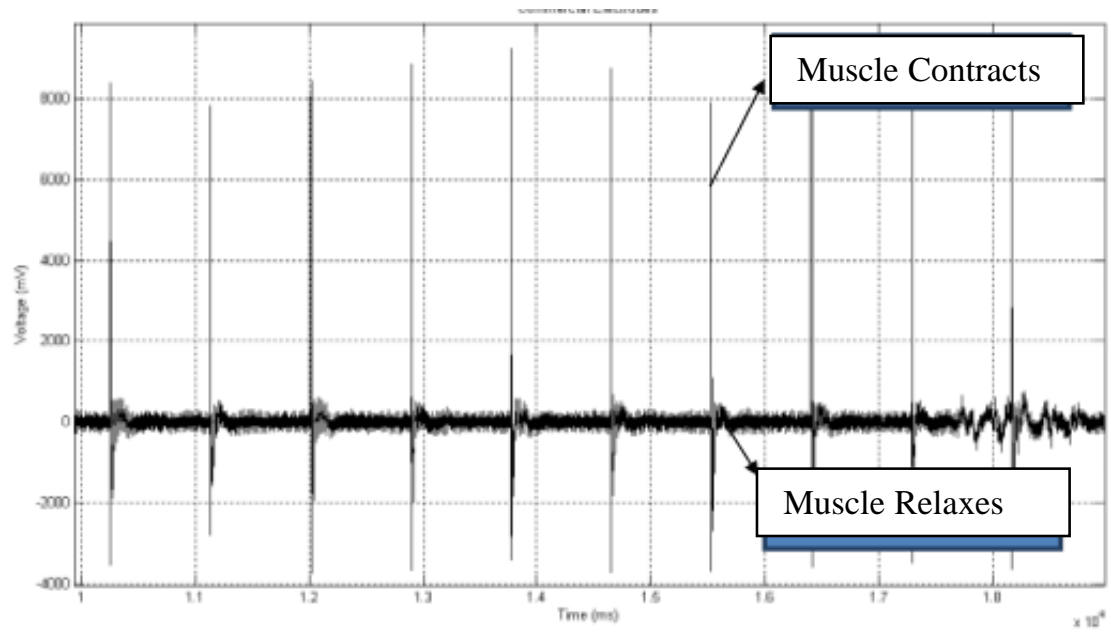

Figure 7. MMG Signal of Commercial Single Electrode

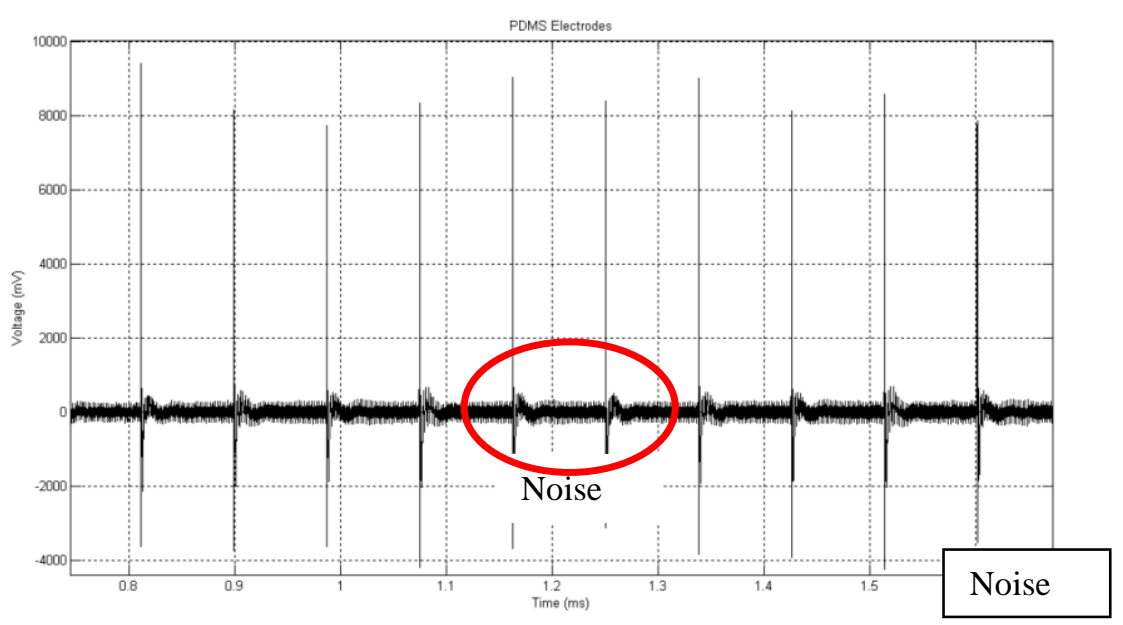

Figure 8. MMG Signal of Ag-PDMS single electrode 


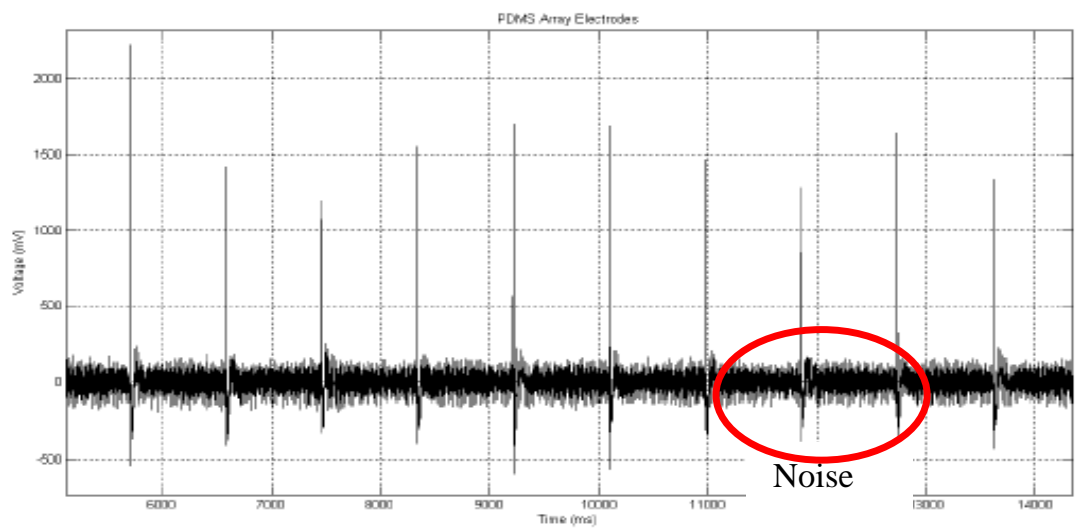

Figure 9. MMG Signal of Ag-PDMS array electrode

\section{Conclusion}

The paper presents the development and characterization of Ag-PDMS and Ag on OHP film electrodes. The resistivity of Ag- PDMS electrode is higher than Ag-OHP film which means the conductivity of Ag-PDMS electrode is lower than Ag-OHP film electrode. This is due to the presence of PDMS polymer in the Ag paste which will reduce the conductivity in the composite. The resistivity difference of the composite due to mechanical force is essential and could affect the electrical properties of a flexible circuit. Results show that the resistivity of the Ag-PDMS composite increases after the bending and higher when compared to Ag paste.

Functionality tests have proven that the Ag-PDMS single electrode is comparable to the commercial electrode showing similar results of measured MMG signals. Between single electrode and array electrode, results indicate that the MMG peak signal of single electrode is much higher than array electrode for both substrates. All in all, it can be said that both of the substrates have their own advantage and disadvantage. Both substrates with both designs are well-functioned. However, in terms of the adhesion and conductivity, the Ag-OHP paper electrode gives the better result than Ag-PDMS electrode but not without limitation. The former could only be bent but the latter has the ability to be stretched and bent. These results also have make evident that screen printing can be used to develop low cost Ag-PDMS electrode which can be implemented in other wearable electrode areas.

\section{References}

[1] Huang GW, Xiao HM, Fu SY. Wearable electronics of silver-nanowire/poly (dimethylsiloxane) nanocomposite for smart clothing. Scientific reports. 2015;5.

[2] Bhore SS. Formulation and Evaluation of Resistive Inks for Applications in Printed Electronics. Retrieved from http://scholarworks.wmich.edu/masters_theses

[3] Niu XZ, Peng SL, Liu LY, Wen WJ, Sheng P. Characterizing and patterning of PDMS based conducting composites. Advanced Materials. 2007; 19(18): 2682-6. http://doi.org/10.1002/adma.200602515

[4] Yuan W, Gu W, Lin J, Cui Z. Printed flexible and stretchable hybrid electronic systems for wearable applications. In Electronic System-Integration Technology Conference (ESTC). 2016: 1-4.

[5] Larmagnac A, Eggenberger S, Janossy H, Vörös J. Stretchable electronics based on Ag-PDMS composites. Scientific reports. 2014; 4.

[6] Wang Z. Polydimethylsiloxane mechanical properties measured by macroscopic compression and nanoindentation techniques. University of South Florida. 2011.

[7] Chen Sc, Yu Ch, Liu Cl, Kuo Ch, Hsu St. Design of surface electrode array applied for hand functional electrical stimulation in the variation of forearm gesture. 2007; 24(23): 24.

[8] Guo H, Tang J, Zhao M, Zhang W, Yang J, Zhang B, Chou X, Liu J, Xue C, Zhang W. Highly stretchable anisotropic structures for flexible micro/nano-electrode applications. Nanoscale research letters. 2016; 11(1): 112.

[9] Yu HK. Copper Micro-Labyrinth with Graphene Skin: New Transparent Flexible Electrodes with Ultimate Low Sheet Resistivity and Superior Stability. Nanomaterials. 2016; 6(9): 161.

[10] Syed, Amjad Ali, Xing-guang Duan, Arbab Nighat Khizer, Mengli Mengli, Xiangzhan Kong, Qiang Huang. Design and Implementation of Probe Driver Teleoperative Force Feedback System. Indonesian Journal of Electrical Engineering and Computer Science. 2014; 6: 4215-4221. 